\title{
Interoperability as a Property of Ubiquitous Healthcare Systems
}

\author{
Milan Zdravković*. Ovidiu Noran.** \\ Miroslav Trajanović*** \\ *LIPS, Faculty of Mechanical Engineering in Niš, University of Niš, \\ Serbia (e-mail:milan.zdravkovic@masfak.ni.ac.rs). \\ **Centre for Enterprise Architecture Research and Management (CEARM), \\ Griffith University, Australia (e-mail:o.noran@griffith.edu.au) \\ ***LIPS, Faculty of Mechanical Engineering in Niš, University of Niš, \\ Serbia (e-mail:miroslav.trajanovic@masfak.ni.ac.rs).
}

\begin{abstract}
As healthcare becomes omnipresent, the contemporary paradigm of systems interoperability turns out to be incomplete and insufficient in attempt to address the complex interrelationships of diversified technical environment in which the clinical processes occur. While the traditional view of the healthcare systems architecture considered only clinical information systems and healthcare facilities, as the future Internet-of-Things becomes a reality, mobile devices, sensors, tags and other identifiable resources with communication and processing capability need to be taken in the picture. In such complex circumstances, the perception of interoperability needs to evolve from the consideration of interoperating pairs of systems to the capability of an autonomous system to sense, interpret, understand and act upon arbitrary messages received from a potentially unknown sender. In this sense, interoperability becomes in fact a property of the system. In this paper, we elaborate on an evolved concept of interoperability in context of ubiquitous healthcare systems. Then, we identify the enabling factors for the interoperable systems involved in pervasive healthcare and assess the impact the Interoperability as a Property (IaaP) paradigm would have on the healthcare landscape and challenges.
\end{abstract}

\section{INTRODUCTION}

The motivation for this work arises from two major issues present in today's healthcare landscape. First, there is a notable lack of capacity in the clinical centres, especially when aspects of prevention and early detection are considered. This lack of capacity is becoming critical in the context of ongoing demographic changes in the world. Thus, population aging, while a positive fact reflecting increased longevity and eradication of some serious diseases, has unfortunately also resulted in significant social security and healthcare challenges (International Health Organisation, 2009). The aged, children and chronically ill need continuous monitoring and care, which brings about the growing issue of overloaded clinical environments where many hospitals nowadays often operate at, or over capacity. A possible solution proposed by Ko et al. (2010) involves the shift from a centralized, expert-driven, crisis-care model to one based on prevention and early detection, implemented both in homes and clinical centres. This however, requires true cooperation of healthcare providers, which is a nontrivial issue. Cooperation is also paramount in healthcare emergency situations such as pandemics whose occurrence appears to be on the rise and increasingly involve drugresistant strains (Waugh and Streib, 2006).

Thousands of patients die each year in hospitals due to medical errors, largely preventable by effective collaboration and continuous monitoring (Nembhard and Edmondson,
2006). This brings about the second major issue identified in the healthcare domain: in the process of diagnosis and treatments the observations are often biased due to the lack of, or restricted information access to a wider context of patient behaviour and lifestyle. Thus, current medical diagnostic facilities are typically insensitive to the context of the measurements or diagnostic actions, resulting in potentially biased observations - especially when unusual observation data patterns are considered. This problem is also present at a wider social scale of biomedical research; thus, for example human behaviour studies are difficult to perform in laboratory environment, where the complexity of human life cannot be properly recreated. The alternative, selfreporting methods suffer from bias and errors.

The paradigm of pervasive or ubiquitous computing aims to address the above-mentioned challenges. Thus, ubiquitous computing aims to provide a more natural interaction of the humans with information and services, by embedding specific artefacts into their environment as unobtrusively as possible (Estrin et al, 2002). An important aspect however, is that the devices that interact with humans and among themselves must be aware of the context. The emergence of pervasive internet connectivity makes this possible and provides high expectations for the so-called Internet-ofThings (IoT) paradigm. Note that in human-centred ubiquitous computing, humans are seen as 'nodes' assimilated into the network. While beneficial from an integration point of view, this perspective largely ignores human-specific aspects. 
One of the greatest challenges for the IoT is making different devices exchange the relevant information and consequently, making them interoperate. ISO/IEC 2382 vocabulary for information technology defines interoperability as "the capability to communicate, execute programs, or transfer data among various functional units in a manner that requires the user to have little or no knowledge of the unique characteristics of those units". In a more broad sense, IEEE defines interoperability as "the ability of two or more systems or components to exchange information and to use the information that has been exchanged" (IEEE, 1990). Here, interoperability means that systems must negotiate 'on the fly' in order to interoperate - no pre-determined assets for interoperations are assumed. ISO14258 (ISO, 2005) defines three main types of interoperability: 'integration' (total interoperability), 'federalisation' (minimal interoperability, hence mere compatibility) and 'unified', where ontology is negotiated in advance so as to ensure semantic interoperability.

In light of the requirements of the future IoT, we identify key problems with the current definitions of interoperability. They assume necessary awareness and agreement of both actors about their behaviours for a given interaction. This assumption is derived from the predefined motivation to interoperate. In fact, they assume awareness of the coexistence of two systems that interoperate.

Unfortunately however, such assumptions cannot hold in future ad-hoc communication and interoperation of the anticipated vast variety of systems participating in ubiquitous computing. Even though the current collaboration culture assumes sharing and a social context, these may become obstacles for interoperability because they imply previous agreements between the interoperating systems. Removing these agreements would mean that interoperability will become, in fact, a semantic issue. This can be dealt with from an anthropomorphic perspective, where the systems can sense, observe, perceive and if necessary, act. Thus, interoperability can become the property of a single system, reflecting its capability to communicate like humans.

The key research questions behind the work presented in this paper are: 1) what is needed to enable the interoperability as a property (IaaP) and 2) how this affects the healthcare landscape.

\section{UBIQUITOUS COMPUTING IN HEALTHCARE}

Ubiquitous healthcare supported by an infrastructure relying (among others) on Wireless Sensor Networks (WSNs) and pervasive internet connectivity have emerged in recent years.

Medical sensors combine transducers for spatial-temporal detection of electrical, thermal, optical, chemical, genetic and other signals with physiological origin with signal processing algorithms to estimate features indicative of a person's health status (Ko et al, 2010). Besides physiological data, the sensors also collect environment and logistics data (e.g. patients' locations, equipment locations), needed, not only for detection, diagnosis and treatment of medical symptoms, but also for the management of a clinical workflow in which these activities occur. In fact, combining different types of information from the different types of sensors facilitates context-awareness.

Besides sensing their environment, embedded and implantable sensors are being now used to trigger physiological and other interventions (e.g., brain-controlled motor prosthetics or preventing epileptic seizures). Hence, medical sensors today can be considered as systems, which, beside transmitters and processing units also host actuators. Types of the medical sensors are: thermometers, blood pressure monitors, glucose monitors (glucometers), electrocardiograms (ECG), photoplethysmogram (PPG), electroencephalography (EEG), imaging sensors, accelerometers, gyroscopes, GPS devices. Environmental sensors for providing contextual information are: RFID readers, video cameras, sound, pressure, temperature, luminosity and humidity sensors. Different types of actuators can be associated to the sensor node, such as: device triggers, alarms, pacemakers, insulin pumps, etc.

Today, there are many different IoT applications that could significantly change the landscape of healthcare, particularly in aspects of monitoring and prevention, clinical workflow efficiency and wider-scale clinical researches.

Home-based, real-time health-related monitoring of people behaviour, particularly for certain demographics can significantly improve their life quality (Patrick, 2009). The related observations can be even correlated with a social and environmental (e.g. exposure to environmental factors, such as pollution) context to acquire more relevant and more useful observations in order to make the most informed conclusions. Healthcare IoT systems are today complemented by IoT applications in other domains. For example, smart homes with context-aware infrared sensors, computers, biosensors and video cameras, emergency communication, control of home appliances, acoustic tracking (Liao et al, 2005) are the environments which per se can be used for monitoring the people behaviour, at least in homes. The examples of more specific applications are assisting devices for the visually impaired, such as way finding and walking navigation (Dabiri et al, 2008) and sensors integrated into clothing which detects biochemical changes in sweat, which may indicate some health related problems, even in real-time (Morris et al, 2009). High resolution monitoring of movement and activity levels can be used for the purpose of recovering patient's motor coordination (used to measure the effect of treatments) or in case of a need for continuous monitoring for cognitive disorders, such as Alzheimer's and Parkinson's. There are systems that collect user activity data to characterize certain patterns, such as "walking", "sitting" or "typing" (Ganti et al, 2006), or that can detect certain postures or falls, even before incident occurs (Nyan et al, 2008).

Clinical real-time patient monitoring systems are implemented for the purpose of early detection of clinical emergency. They are used to continuously track the vital signs of the patient, e.g. pulse oximetry, respiration rate, temperature, heart rate, heart rate variability, arterial blood pressure, skin temperature, skin conductance, blood alcohol 
concentration. Besides the vital signs information, the typical factors of the response to this clinical emergency are the patient's EHR and assignment of the respondent (doctor), based on physical location information (Rastegari et al, 2011). Hence, location and proximity sensing technologies can have significant effect in improving the workflow efficiency in hospitals (Fry and Lenert, 2005). Often, physical location of a person or an object is a very important factor for the decisions that need to be very fast and reliable. For example, in case of a critical condition of a patient, it is important to identify the nearest doctor. In another example, IoT technologies can be used for automatic triage of patients for providing emergency care in large disasters, including tracking the health status of the first respondents (Gao et al, 2008).

Synthesis of data acquired from the potentially large number of sensors is also useful for facilitating large scale field studies of human behaviour and chronic diseases, for example to track the spread of diseases by public health agencies (Hanjagi et al, 2007) or to research the use of energy during different activities and the variance across a population of subjects (Patrick, 2009). This type of applications combine body-area wireless sensor networks with sensor-equipped smart phones and cloud-based data storage and processing services, leading to a new paradigm of population-scale medical research studies.

Still, there are many problems that need to be resolved in the future. The technical issues of WSNs, such as limited network capacity, processing and memory constraints, energy constraints, issues of system reliability (e.g. in data delivery over multi-hop WSNs) and related variance in quality of service shows that this technology is still immature.

Accuracy of data and respective trustworthiness of the healthcare WSN infrastructure can be considered in the aspects of their integrity and availability. Ko et al (2009) found that the rate of packet losses for radios based on IEEE 802.15.4 standard is much higher in hospitals than in other indoor environments. The source of this issue is concentration of the devices with which there is a risk of interferences, such as Wi-Fi networks, Bluetooth devices, cordless phones and similar devices. The aspect of availability is related to a latency issue. Delivery latency is important for the systems with actuators, especially when urgent action is required. Although pervasive systems facilitate continuous tracking and monitoring, they are in fact soft real-time systems, where some latency is allowed (Shin and Ramanathan, 1994).

\section{INTEROPERABILITY AS A PROPERTY}

Current use cases for the future IoT are typically based on pre-agreements of the various devices to exchange information and to act upon this information. However, as the number of connected devices and their technological diversity grows, it would become more and more difficult to work on reaching these pre-agreements. In addition, the current approach will inevitably lead to application silos, with fragmented architectures, incoherent unifying concepts and hence, little reuse potential. This fragmentation, which occurs due to the restricted domain of interest of the different architectures, is a barrier to context awareness, leading to potentially biased observations. For example, diastolic blood pressure increases significantly at the lower temperatures of the environment in which this pressure is observed. When the information about the temperature factor is not available, this can lead to false conclusions.

Thus, it is highly likely that the 'things' belonging to the future IoT will be required to interpret ad-hoc signals and requests from other devices, interpret their meaning and act accordingly. For example, even if the room temperature is not a feature of interest for a blood pressure measuring device, the latter will observe and perceive this information while interacting with surrounding temperature sensors (whatever and wherever these sensors may be) and take it into account in the processing, during the continuous monitoring process.

To demonstrate the complexity of the ubiquitous environments, we will refer to the control theory. Namely, any 'thing' in the IoT can be considered a closed-loop, or feedback control system. To implement a process control, a controller must collect data from and transmit feedback to this process. The process control is carried out by actuators, which deliver continuous and discrete parameters to the process, based on the processed continuous and discrete variables. The typical objective of the process control is to maintain a stable operation, namely a set point, by calculating and issuing solutions for the appropriate corrective actions.

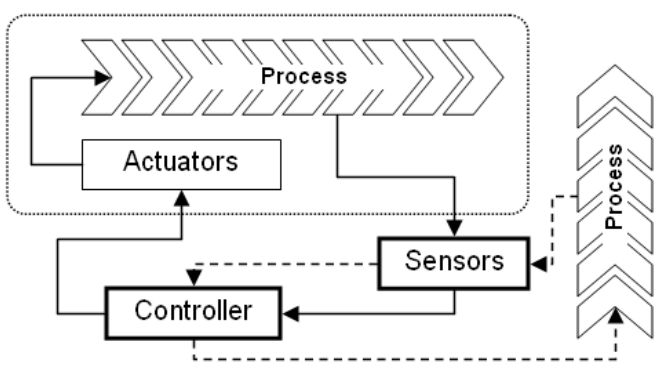

Fig. 1. Extended control system

In the interoperable world of ubiquitous computing, it is assumed that the roles of sensors and controllers would not be exclusive to the process to which they are initially committed. Thus sensors are measuring continuous and discrete variables for the processes in their environment, while controllers interpret these variables and may decide on corresponding actions on other processes (see Fig.1). Thus, new roles (in fact, new processes) may be mandated to the sensor and controller components of the control system. However, this is only a functional boundary as the sensor, controller and actuator remain within their physical unit. Hence, a new requirement for sensors and controllers is that they need to be able to receive and process new types of signals, possibly unrelated to the process they were initially committed to.

In order for this to become possible, sensors and controllers need to be capable to interpret these signals, infer their meanings and infer the action(s) to carry out based on these meanings (but not necessarily to perform those actions). This 
capability is required in order to be able to interoperate in the environment of ubiquitous systems - be it information systems (IS), software agents or devices.

Let us consider a scenario of future IoT in which a person with embedded blood pressure sensor is walking on the street (or is moving / being moved between hospitals/ hospital departments) (see Fig.2). This sensor $\left(\mathrm{N}_{1}\right)$ is capable to sense and perceive any message, received from its environment.

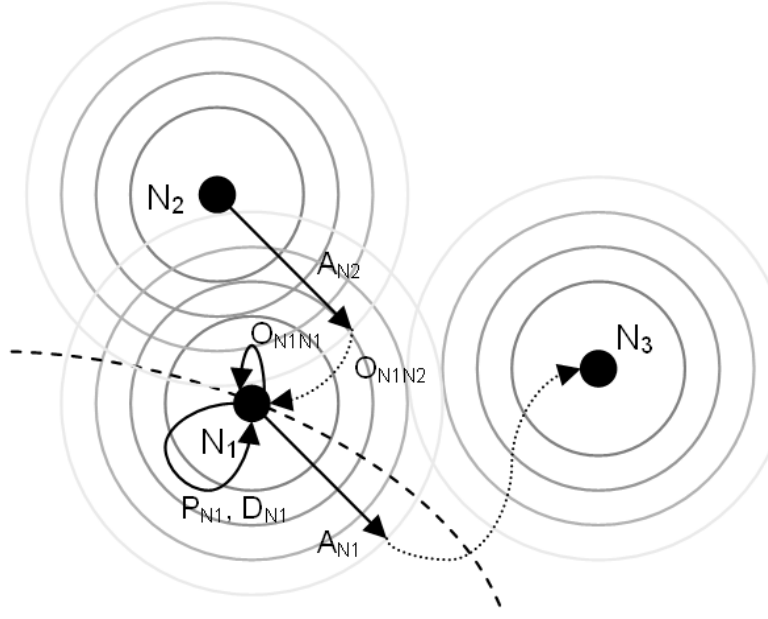

Fig. 2. Scenario of future IoT

In the environment of $\mathrm{N}_{1}$, there are other sensors, observing the environment and continuously transmitting the observed data. For example, temperature sensor $\mathrm{N}_{2}$ is continuously sending message $A_{N 2}$, with air temperature. This message is sensed and observed $\left(\mathrm{O}_{\mathrm{N} 1 \mathrm{~N} 2}\right)$ by $\mathrm{N}_{1}$. In the meantime, blood pressure sensor is continuously collecting its own observations $\left(\mathrm{O}_{\mathrm{N} 1 \mathrm{~N} 1}\right)$. As it was mentioned before, perception of the blood pressure raise, without consideration of the temperature of the environment can lead to false conclusions. Actually, in this case, $\mathrm{N}_{1}$ is creating a percept $\mathrm{P}_{1}$, based on two observations $\mathrm{O}_{\mathrm{N} 1 \mathrm{~N} 2}$ and $\mathrm{O}_{\mathrm{N} 1 \mathrm{~N} 1}$. Now, based on this perception, $\mathrm{N}_{1}$ is capable to make a decision $\mathrm{D}_{1}$, e.g. to send SMS to a physician. Hence, $\mathrm{N}_{1}$ articulates and sends out a message $A_{N 1}$, with request to send SMS with designated content and recipient. Finally, there is a device $\mathrm{N}_{3}$ with SMS sending capability, which observes this message and acts further upon it.

\section{ENABLING FACTORS FOR IAAP}

Let us refer to the first research question: what is needed to make the above scenario possible; what are the enabling factors for interoperability as a property?

Adopting an anthropomorphic stance and using the typical scenario in Fig. 2, we can set the basic requirements for the autonomous, intelligent, purposeful and social behaviour of a 'thing' in an interoperable environment (such as WSN). Thus, a thing exhibiting interoperability as a property must feature awareness, perceptivity, intelligence and extroversion.

In regards to awareness, we can distinguish two aspects: selfawareness and environmental awareness. Self-awareness is related to the capability of the thing to sense a phenomenon or an event within itself. For example, WSN nodes need to be aware of the available energy levels. Environmental awareness is related to the capability of the thing to sense a phenomenon or an event from its environment, extended by the capability to receive a message from its environment. It is important to highlight that currently, the awareness of nodes is functional in its nature and thus, restricted; namely, the sensor is aware only of the environmental features matching its pre-determined interest. A similar point can be made related to the capability of the thing to receive a message of a known format. Hence, we can also further distinguish between functional and universal environmental awareness.

Perceptivity is the property of a thing related to the capability to assign a meaning to an observation. Note that observations can occur within itself or from its environment and that they are typically multi-modal (e.g. temperature, light, sound, etc.) and possibly multi- dimensional (e.g. they may be time and location dependent). While awareness and self-awareness are properties that have been already achieved by WSN nodes, they have occurred only in the restricted, functional scope; perceptivity goes one step further by facilitating universal awareness. Perceptivity enables things to observe based on arbitrary stimuli and interpret these observations, transforming the physical observations into a meaningful percept. Based on this perception, the thing should be able to decide on the consequent action.

The decision to act based on a perception should be the result of a cognitive process, consisting of identification, analysis and synthesis of the possible actions to perform in response to the understood observation (i.e. the percept). Therefore, interoperability as a property must feature intelligence encompassing assertion, storing and acquisition of the behaviour patterns, based on the post-agreements in regards to the purposefulness of the performed actions.

Another required attribute of the thing would be extroversion, related to the willingness and capability of the thing to articulate its above actions. This attribute demonstrates the thing's concern about its physical and social environment. It also reflects 'curiosity' as the capability to articulate the request for additional information needed for a complete reasoning during the processes of perception and decision.

\section{UBIQUITOUS, INTEROPERABLE HEALTHCARE SYSTEMS}

This section deals with the second research question, namely how would the IaaP paradigm impact on current healthcare situation and assist healthcare providers in meeting the significant challenges that lay ahead. Organisational and technical perspectives are provided.

When dealing with complex systems such as healthcare organisations, interoperability has many facets, as described in several mainstream interoperability frameworks. However, the combined framework analysis performed by Noran and Panetto (2013) has shown that even at this level, semantic interoperability appears to still prevail as the most important and difficult to solve aspect. This encourages the extension of the proposed IaaP concept to healthcare providers as a whole. 
To start with, extending the IaaP concept to the entire organisation would assist healthcare providers in gaining agility. An agile organisation would be able to interoperate to a large(r) degree without having to become integrated in a specific negotiated framework or system / of systems (ibid.). Preserving organisation independence and resilience which would prove crucial in emergency situations where task force partners may unexpectedly fail, with the rest of the team having to 'cover' the failed partner's functions or promptly find a replacement. 'Taking over' another organisation's tasks may require prompt interoperation in areas not previously negotiated and thus facilitated by displaying IaaP.

Healthcare providers and their information systems are highly heterogeneous and hierarchical, posing a variety of internal and external interoperability barriers as described by Noran and Panetto (2013). The IaaP concept can be used to alleviate or even overcome some effects of these barriers. Thus, as further detailed, internal information sharing (disrupted by hierarchy and culture when performed by humans) could be greatly improved. Externally, by displaying IaaP, healthcare providers could significantly reduce the time required to set up task forces for short term medical emergencies and to take part in 'healthcare management virtual organisations' (Noran, 2013) for long term problems such as increased demand due to population aging and the need for care at home for chronically ill etc. These beneficial effects are further explained below in relation to each of the previously defined IaaP enabling factors.

Awareness. True and efficient collaboration is not possible unless the organisational cultures, processes and resources of the participants possess the required interoperability preparedness (Kapucu et al., 2010). Universal environmental awareness would greatly enhance the healthcare provider's preparedness for cooperation, both inside and outside its own boundaries. Thus, on the internal level medical safety and collaboration between various departments of healthcare providers would be dramatically improved as monitoring devices would keep track of the inpatients and be able to interoperate across the healthcare provider's departments. Thus, the lack of interoperability of the current systems (some of which do not satisfy even the compatibility requirement, requiring staff to manually transfer data) would be replaced by ubiquitous awareness and data sharing for every patient. On the external level, the healthcare provider would be able to seamlessly exchange information with other healthcare providers about common patients and also monitor outpatients' recovery progress in real time, irrespective of location and taking into account all relevant ambient factors.

Perceptivity and Intelligence. All healthcare organisations implement some kind of knowledge management system / business intelligence; however, typically they only cover the upper and possibly middle management levels. In the IaaP scenario, such a knowledge management system would evolve into an expert system extending throughout the organisation, from top management to the real-time response units, even medical devices, enabled by the pervasive, ubiquitous computing framework and intelligent sensors and controllers. The healthcare provider would become a learning organisation and constantly improve and adjust its response to external challenges - thus increasing its agility.

Extroversion. The social effect of an extrovert healthcare system (e.g. manifested by transparency towards patients, other providers and general public) would be tremendously positive; especially in large scale healthcare incidents, trust and communication are paramount in an effective response and minimising negative effects.

The IaaP paradigm and its enabling factors would also benefit the technical aspect of IoT by resolving some of the issues specific to its application in healthcare.

Thus, the reliability issue present due to the extreme environmental conditions that affect medical sensors' communication is currently addressed by mostly technical approaches, such as redundancy (Chipara et al, 2010). The IaaP concept reduces the reliability problem to ensuring completeness and correctness of reasoning during the perception process. Hence, reliability can be addressed by perceptual sets (typically employing various models and meta-models) used by the things to perceive the observations.

A similar approach can be used to resolve potential privacy and security problems. Instead of using various conventional and non-conventional authentication schemes, the messages and signals emitted by the things would include formal descriptions of the privacy and security policies. Such policies would then become part of the perceptual sets by providing additional context for decision-making in relation to potential consequent action.

Finally, the energy consumption issue is also addressed by this approach. Although the concept of IaaP potentially implies more traffic between the things in IoT, such an increase would be compensated by the intelligent processing capability. For example, in multi-hop WSNs, perceiving raw sensor data, interpreting it and transmitting the resulting meaningful percept (or acting upon it) as opposed to simply passing this raw data can significantly reduce the volume of data that needs to be communicated from the sensor nodes to the gateways or processing components. Allocating a processing capability to things may in fact reduce the number of components, with a similar effect on the traffic.

\section{CONCLUSIONS AND FURTHER WORK}

The current understanding of the interoperability concept is being challenged by new technologies and paradigms. After an evaluation of the current healthcare problem domain and a brief review of the ubiquitous computing state-of-the-art in healthcare, we have alerted to the impending interoperability conundrum posed by the exploding number of devices ('things") that populate the IoT. As a possible solution, we have proposed a new paradigm describing interoperability as a property of every system aspiring to efficiently interoperate (and thus survive) in the future IoT. Next, we have defined the enabling factors in the evolution of interoperability from a typical set of agreements shared between several interoperating parties to a property owned by a single system. Finally, we have investigated the applicability of the concept 
to a larger scale and the changes that 'interoperability as a property' may bring to the current healthcare scenario.

Further work is required to test and refine the IaaP concept and to tackle several outstanding issues. Thus, the relevance and impact of IaaP on cultural interoperability (Whitman and Panetto, 2006) and trust, aspects specific to the human component of the healthcare systems, must be further clarified. Furthermore, the influence of the life cycle phase of the system of interest on its capacity to display IaaP must also be taken more into account.

\section{REFERENCES}

Ko, J., Lu, C., Srivastava, M.B., Stankovic, J.A., Terzis, A., Welsh, M. (2010) Wireless sensor networks for healthcare. Proceedings of the IEEE. Vol.98, Issue 11

Estrin, D., Culler, D., Pister, K., Sukhatme, G. (2002). Connecting the Physical World with Pervasive Networks. IEEE Pervasive Computing. Vol.1, Issue 1, pp 59-69

Patrick, K. (2009) A tool for geospatial analysis of physical activity: Physical activity location measurement system (PALMS). Medicine and Science in Sports and Exercise. Vol.41, Issue 5.

Liao, L., Fox, D., Kautz, H. (2005) Location-based activity recognition using relational Markov networks. In: Proceedings of the 19th international joint conference on Artificial intelligence. pp 773-778. Morgan Kaufmann Publishers Inc. San Francisco, CA, USA

Dabiri, F., Vahdatpour, A., Noshadi, H., Hagopian, H., Sarrafzadeh, M. (2008) Ubiquitous personal assistive system for neuropathy. In: Proceedings of the $2^{\text {nd }}$ International Workshop on Systems and Networking Support for Health Care and Assisted Living Environments. Article No. 17. ACM New York, NY, USA

Morris, D., Coyle, S., Wu, Y., Lau, K.T., Wallace, G., Diamond, D. (2009) Bio-sensing textile based patch with integrated optical detection system for sweat monitoring. Sensors and Actuators B: Chemical. Vol.139, Issue 1, pp. 231-236

Ganti, R.K., Jayachandran, P., Abdelzaher, T.F., Stankovic, J.A. (2006) SATIRE: A software architecture for smart AtTIRE. In: Proceedings of the 4th international conference on Mobile systems, applications and services. pp. 110-123. ACM New York, NY, USA

Nyan, M.N., Tay, F.E.H., Murugasu, E. (2008) A wearable system for pre-impact fall detection. Journal of Biomechanics. Vol.41, Issue 16, pp. 3475-3481

Rastegari, E., Rahmani, A., Setayeshi, S. (2011) Pervasive computing in healthcare systems. World Academy of Science, Engineering and Technology. Vol.2011, Issue 59

Fry, E.A., Lenert, L.A. (2005) MASCAL: RFID tracking of patients, staff and equipment to enhance hospital response to mass casualty events. In: AMIA Annual Symposium Proceedings Archive. Vol.2005, pp.261-265.

Gao, T., Pesto, C., Selavo, L., Chen, Y., Ko, J., Lim, J.H., Terzis, A., Watt, A., Jeng, J., Chen, B., Lorincz, K., Welsh, M. (2008) Wireless medical sensor networks in emergency response: Implementation and pilot results. In: Proceedings of IEEE Conference on Technologies for Homeland Security. pp.187 - 192

Hanjagi, A., Srihari, P., Rayamane, A.S. (2007) A public healthcare information system using GIS and GPS: A case study of Shiggaon. GIS for Health and the Environment: Lecture Notes in Geoinformation and Cartography. Vol.2007, pp.243-255. Springer Berlin Heidelberg

Ko, J., Gao, T., Terzis, A. (2009) Empirical study of medical sensor application in an urban emergency environment. In: Proceedings of the Fourth International Conference on Body Area Networks. Article No. 10

Shin, K.G., Ramanathan, P. (1994) Real-time computing: a new discipline of computer science and engineering. Proceedings of the IEEE. Vol.82, Issue: 1

IEEE (1990). "IEEE Standard Computer Dictionary: A Compilation of IEEE Standard Computer Glossaries", Institute of Electrical and Electronics Engineers, 1990

Noran, O., Panetto, H. (2013) Modelling a Sustainable Cooperative Healthcare: An Interoperability-driven Approach. Lecture Notes in Computer Science. Vol.8186, pp.238-249

International Labour Organisation. (2009) Ageing societies: The benefits, and the costs, of living longer. (Accessed 2013 at http://www.ilo.org/global/WCM_041965/lang-en/index.htm.)

Noran, O. (2013) Enhancing Collaborative Healthcare Synergy. IFIP Advances in Information and Communication Technology. Vol.408, pp.459-467

Waugh, W.L., Streib, G. (2006) Collaboration and Leadership for Effective Emergency Management. Public Administration Review. Vol.66/s1, pp. 131-140

International Standardisation Organisation (2005). ISO14258: Industrial Automation Systems, Concepts and Rules for Enterprise Models.

Whitman, L., Panetto, H. (2006) The Missing Link: Culture and Language Barriers to Interoperability. Annual Reviews in Control, Vol.30, Issue 2, pp. 233-241

Kapucu, N., Arslan, T., Demiroz, F. (2010) Collaborative emergency management and national emergency management network. Disaster Prevention and Management. Vol.19, Issue 4, pp. 452-468

Nembhard, I.M., A.C. Edmondson (2006) Making It Safe: The Effects of Leader Inclusiveness and Professional Status on Psychological Safety and Improvement Efforts in Health Care Teams. Journal of Organizational Behavior. Vol.27, Issue 7, pp.941-966

Chipara, O., Lu, C., Bailey, T.C., Roman, G.C. (2010) Reliable clinical monitoring using wireless sensor networks: experiences in a step-down hospital unit. In: Proceedings of the 8th ACM Conference on Embedded Networked Sensor Systems, pp.155-168, ACM New York, NY, USA 